\title{
Dirac $\boldsymbol{R}$-matrix calculations of electron-impact excitation of neon-like krypton
}

\author{
D C Griffin ${ }^{1}$, C P Ballance ${ }^{1}$, D M Mitnik ${ }^{2}$ and J C Berengut ${ }^{3}$ \\ ${ }^{1}$ Department of Physics, Rollins College, Winter Park, FL 32789, USA \\ ${ }^{2}$ Instituto de Astronomía y Física del Espacio, and Departamento de Física, Universidad de Buenos \\ Aires, Argentina \\ ${ }^{3}$ School of Physics, University of New South Wales, Sydney 2052, Australia
}

Received 5 August 2008, in final form 4 September 2008

Published 13 October 2008

Online at stacks.iop.org/JPhysB/41/215201

\begin{abstract}
We have employed the Dirac $R$-matrix method to determine electron-impact excitation cross sections and effective collision strengths in Ne-like $\mathrm{Kr}^{26+}$. Both the configuration-interaction expansion of the target and the close-coupling expansion employed in the scattering calculation included 139 levels up through $n=5$. Many of the cross sections are found to exhibit very strong resonances, yet the effects of radiation damping on the resonance contributions are relatively small. Using these collisional data along with multi-configuration Dirac-Fock radiative rates, we have performed collisional-radiative modeling calculations to determine line-intensity ratios for various radiative transitions that have been employed for diagnostics of other Ne-like ions.
\end{abstract}

(Some figures in this article are in colour only in the electronic version)

\section{Introduction}

Neon-like ions play an important role in the diagnostics of a wide variety of laboratory and astrophysical plasmas. Primarily because of its importance to astrophysical plasmas, one of the most thoroughly studied neon-like ions is $\mathrm{Fe}^{16+}$. For this ion, there has been a long series of distortedwave (DW) calculations that include the effects of resonant recombination followed by autoionization [1-4] as well as Breit-Pauli (BP) $R$-matrix [5, 6] and Dirac $R$-matrix [7, 8] calculations of electron-impact excitation. Neon-like krypton is also important, especially as a diagnostic for tokamak plasmas [9]. Although there was some early work on electron-impact excitation of $\mathrm{Kr}^{26+}$ using the DW [10], the relativistic DW [11] and Coulomb-Born [12] approximations, the only published $R$-matrix calculation is a 27 -level BP calculation by Gupta et al [13]. In addition, Rice et al [9] carried out extensive calculations of the energy levels, radiative rates and electron-impact cross sections for $\mathrm{Ne}$ like krypton, zirconium, niobium and molybdenum. They employed these data for calculating theoretical spectra and compared them with observed spectra from tokamak plasmas.
Their excitation cross sections were determined in the relativistic DW approximation; however, it is not clear that the contributions from resonant recombination followed by autoionization were included. These contributions are always present in $R$-matrix calculations.

In this paper, we report on the results of a 139-level Dirac $R$-matrix calculation of electron-impact excitation in $\mathrm{Kr}^{26+}$. With resonant contributions to excitation, certain types of radiative rates from recombination resonances increase with ionization stage, while autoionizing rates remain relatively constant. Radiative decay of these resonant states to the bound states of the recombined atom, which is referred to as radiative damping, can reduce the size of these resonances. We have investigated these effects in $\mathrm{Kr}^{26+}$ by performing two $R$-matrix calculations-one with radiative damping and one without. By comparing the two results, we are able to assess the importance of damping on the electron-impact excitation of this ion. Finally, we incorporated our calculated radiative and collisional rates in collisional-radiative calculations of lineintensity ratios for a set of transitions that have been employed in the diagnostics of plasmas for other neon-like ions. 
The remainder of this paper is organized as follows. In the next section, we describe the calculation of the target wavefunctions and present results for energy levels and oscillator strengths. In section 3 , we describe the scattering calculations and present results for excitation cross sections and effective collision strengths as well as line-intensity ratios calculated from these collisional and radiative data. Finally, in section 4, we summarize our findings.

\section{The target structure}

The target orbitals, energy levels, radiative rates and oscillator strengths for this study were generated using the multiconfiguration Dirac-Fock (MCDF) atomic structure program GRASP0 [14, 15]. We included all 139 levels arising from the configurations $2 \mathrm{~s}^{2} 2 \mathrm{p}^{5} n l$ with $n \leqslant 5$ and $2 \mathrm{~s}^{2} \mathrm{p}^{6} n l$ with $n \leqslant 4$ in both the configuration-interaction (CI) expansion of the target and the close-coupling (CC) expansion of the subsequent scattering calculations. The theoretical energies from this calculation are given in table 1 in comparison to existing experimental values. We also show the percentage differences between experiment and theory; the average difference for the 38 levels for which there are experimental values is only $0.059 \%$.

In table 2, we present weighted oscillator strengths $(g f)$ calculated in the length gauge for all dipole-allowed transitions to the ground level. We also give the ratios of the length to velocity oscillator strengths and compare the present $g f$ values with those determined by Rice et al [9]. The oscillator strength ratios are relatively close to one except for the transitions from the two $2 s^{2} 2 p^{5} 5 s$ levels. In addition, with just a few exceptions, the present $g f$ values are in good agreement with those from Rice et al [9]. Six transitions in Ne-like ions are used extensively for diagnostics of laboratory and astrophysical plasmas. The theoretical and experimental wavelengths and theoretical radiative rates for these six transitions in $\mathrm{Kr}^{26+}$, along with their standard labels, are given in table 3 .

Recently, there has been an ongoing controversy over the effects of electron correlation on the radiative rates and the electron-impact excitation collision strengths associated with the $3 \mathrm{C}$ and $3 \mathrm{D}$ transitions in $\mathrm{Fe}^{16+}$. For example, Chen $[4,8]$ states that by adding the two-electron promotion from the $2 p$ to $3 \mathrm{~d}$ subshell along with a set of pseudostates to a comparable 139-level calculation in $\mathrm{Fe}^{16+}$, he obtained a radiative rate for the $3 \mathrm{C}$ transition that is reduced by $9 \%$ while the $3 \mathrm{D}$ radiative rate is increased by $4 \%$. In addition, he states that these same correlations bring the electron-impact excitation collision strengths into closer agreement with experimental measurements.

We would have liked to determine whether these same correlations would have similar effects in the present $\mathrm{Kr}^{26+}$ calculation; however, since Chen [4, 8] does not explain how these pseudostates were generated or what types of correlations they represent, this was not possible. In an earlier study by Dong et al [16], the MCDF method was used to perform a systematic study of the effects of electron correlation on the $3 \mathrm{C}$ and 3D transitions in the Ne-like ions $\mathrm{Cr}^{14+}, \mathrm{Fe}^{16+}$, $\mathrm{Ni}^{18+}, \mathrm{Zn}^{20+}, \mathrm{Ge}^{22+}, \mathrm{Se}^{24+}$ and $\mathrm{Kr}^{26+}$. They first included all possible one- and two-electron promotions out of the $2 \mathrm{~s}$ and $2 \mathrm{p}$ subshells of the $1 \mathrm{~s}^{2} 2 \mathrm{~s}^{2} 2 \mathrm{p}^{6}$ ground configuration to the $3 l, 4 l$ and $5 l$ subshells that produce levels with even parity and $J=0$. They then added all possible one- and two-electron promotions out of the $2 s, 2 p$ and $3 d$ subshells of the $1 s^{2} 2 s^{2} 2 p^{5} 3 d$ excited configuration to the $3 l, 4 l$ and $5 l$ subshells that produce levels with odd parity and $J=1$. Applying these two CI expansions to $\mathrm{Fe}^{16+}$, they obtained a weighted oscillator strength for the $3 \mathrm{C}$ transition of 2.33 , which is $6.4 \%$ lower than that from a 139-level calculation [7] without these correlations. For the $3 \mathrm{D}$ transition, they obtained a value of 0.675 , which is $5.6 \%$ higher than that from the same 139-level calculation [7]. For $\mathrm{Kr}^{26+}$, their weighted oscillator strength for the 3C transition was 1.84 , which is $4.7 \%$ lower than the value given in table 2 for this transition, and for the 3D transition, their weighted oscillator strength was 1.56 , only $0.6 \%$ higher than the value for this transition given in table 2 .

In order to provide an independent test of these results, we have performed $\mathrm{CI}$ calculations on the $3 \mathrm{C}$ and $3 \mathrm{D}$ radiative transitions in both $\mathrm{Fe}^{16+}$ and $\mathrm{Kr}^{26+}$ using a program that has been employed previously to calculate energy levels, relativistic shifts and isotope shifts in a variety of atoms [17-19]. First, we solved the Dirac-Fock equations for the $1 s^{2} 2 s^{2} 2 p^{6}$ ground configuration. Then we generated valence and virtual orbitals by diagonalizing the Dirac-Fock operator on a basis set of B-splines and selecting the orbitals of lowest energy. The CI calculation was carried out using all orbitals up to $7 l$ with $l=\mathrm{s}, \mathrm{p}, \mathrm{d}$ and $\mathrm{f}$, which enabled us to reach a converged result. For our final calculations, we allowed all single and double promotions out of both the $2 \mathrm{~s}$ and $2 \mathrm{p}$ subshells to all valence and virtual orbitals to form states of both even and odd parity. In $\mathrm{Fe}^{16+}$ we tested the effects of allowing excitations from the 1s orbital as well, but they were found to make no difference to the accuracy of the radiative rates quoted here. We also investigated the effects of allowing triple promotions up to the $4 l$ subshells, but again they were found to be very small. From these calculations for $\mathrm{Fe}^{16+}$, we obtained a $3 \mathrm{C}$ weighted oscillator strength of 2.31 , and a $3 \mathrm{D}$ weighted oscillator strength of 0.66 ; these values are $0.9 \%$ and $2.2 \%$ lower, respectively, than those obtained by Dong et al. For $\mathrm{Kr}^{26+}$ we obtained a $3 \mathrm{C}$ weighted oscillator strength of 1.85 and a $3 \mathrm{D}$ oscillator strength of 1.53 ; these are $0.5 \%$ higher and $1.9 \%$ lower, respectively, than the values obtained by Dong et al [16] and $4.1 \%$ lower and $1.3 \%$ higher, respectively, than the values given in table 2 .

Thus, from the earlier work of Dong et al [16] and our own large CI calculations, we would expect that these correlations, which are not included in the target states for the scattering calculation, would have relatively small effects on both the radiative rates and the collision strengths reported here.

\section{Scattering calculations}

The scattering calculations were performed using our suite of parallel Dirac $R$-matrix programs [20] which includes modified portions of the DARC programs [21, 22] as well as a set of modified programs from our parallel BP $R$-matrix 
Table 1. Energies in rydbergs for the first 139 levels in $\mathrm{Kr}^{26+}$ relative to the $2 \mathrm{p}^{6}{ }^{6} \mathrm{~S}_{0}$ ground level.

\begin{tabular}{|c|c|c|c|c|c|c|}
\hline No. & Conf. & $\operatorname{Level}(\mathrm{j}-\mathrm{j})$ & Purity & $\begin{array}{l}\mathrm{MCDF}^{\mathrm{a}} \\
\text { energy }\end{array}$ & $\begin{array}{l}\text { Exp. }{ }^{\text {b }} \\
\text { energy }\end{array}$ & $\%$ Diff. \\
\hline 1 & $2 s^{2} 2 p^{6}$ & ${ }^{1} \mathrm{~S}_{0}$ & 0.999 & 0.000 & 0.000 & 0.000 \\
\hline 2 & $2 s^{2} 2 p^{5} 3 s$ & $(3 / 2,1 / 2)_{2}$ & 0.999 & 121.192 & 121.204 & +0.010 \\
\hline 3 & $2 s^{2} 2 p^{5} 3 s$ & $(3 / 2,1 / 2)_{1}$ & 0.998 & 121.426 & 121.441 & +0.013 \\
\hline 4 & $2 s^{2} 2 p^{5} 3 p$ & $(3 / 2,1 / 2)_{1}$ & 0.952 & 124.964 & 124.966 & +0.001 \\
\hline 5 & $2 s^{2} 2 p^{5} 3 p$ & $(3 / 2,1 / 2)_{2}$ & 0.999 & 125.200 & 125.194 & -0.005 \\
\hline 6 & $2 s^{2} 2 p^{5} 3 s$ & $(1 / 2,1 / 2)_{0}$ & 0.999 & 125.303 & 125.284 & -0.015 \\
\hline 7 & $2 s^{2} 2 p^{5} 3 s$ & $(1 / 2,1 / 2)_{1}$ & 0.998 & 125.430 & 125.400 & -0.025 \\
\hline 8 & $2 s^{2} 2 p^{5} 3 p$ & $(3 / 2,3 / 2)_{3}$ & 1.000 & 126.072 & 126.041 & -0.024 \\
\hline 9 & $2 s^{2} 2 p^{5} 3 p$ & $(3 / 2,3 / 2)_{1}$ & 0.951 & 126.100 & 126.084 & -0.013 \\
\hline 10 & $2 s^{2} 2 p^{5} 3 p$ & $(3 / 2,3 / 2)_{2}$ & 1.000 & 126.410 & 126.397 & -0.010 \\
\hline 11 & $2 s^{2} 2 p^{5} 3 p$ & $(3 / 2,3 / 2)_{0}$ & 0.917 & 127.588 & 127.618 & +0.024 \\
\hline 12 & $2 s^{2} 2 p^{5} 3 p$ & $(1 / 2,1 / 2)_{1}$ & 0.997 & 129.225 & 129.175 & -0.039 \\
\hline 13 & $2 s^{2} 2 p^{5} 3 p$ & $(1 / 2,3 / 2)_{1}$ & 0.993 & 130.241 & 130.192 & -0.038 \\
\hline 14 & $2 s^{2} 2 p^{5} 3 p$ & $(1 / 2,3 / 2)_{2}$ & 0.999 & 130.347 & 130.280 & -0.051 \\
\hline 15 & $2 s^{2} 2 p^{5} 3 d$ & $(3 / 2,3 / 2)_{0}$ & 0.998 & 130.745 & 130.694 & -0.039 \\
\hline 16 & $2 s^{2} 2 p^{5} 3 p$ & $(1 / 2,1 / 2)_{0}$ & 0.917 & 130.908 & 130.742 & -0.127 \\
\hline 17 & $2 s^{2} 2 p^{5} 3 d$ & $(3 / 2,3 / 2)_{1}$ & 0.906 & 130.936 & 130.945 & +0.008 \\
\hline 18 & $2 s^{2} 2 p^{5} 3 d$ & $(3 / 2,3 / 2)_{3}$ & 0.998 & 131.232 & 131.173 & -0.045 \\
\hline 19 & $2 s^{2} 2 p^{5} 3 d$ & $(3 / 2,5 / 2)_{2}$ & 0.704 & 131.266 & 131.233 & -0.025 \\
\hline 20 & $2 s^{2} 2 p^{5} 3 d$ & $(3 / 2,5 / 2)_{4}$ & 1.000 & 131.274 & 131.214 & -0.045 \\
\hline 21 & $2 s^{2} 2 p^{5} 3 d$ & $(3 / 2,3 / 2)_{2}$ & 0.708 & 131.496 & 131.445 & -0.039 \\
\hline 22 & $2 s^{2} 2 p^{5} 3 d$ & $(3 / 2,5 / 2)_{3}$ & 0.999 & 131.723 & 131.664 & -0.046 \\
\hline 23 & $2 s^{2} 2 p^{5} 3 d$ & $(3 / 2,5 / 2)_{1}$ & 0.879 & 132.551 & 132.476 & -0.057 \\
\hline 24 & $2 s^{2} 2 p^{5} 3 d$ & $(1 / 2,3 / 2)_{2}$ & 0.998 & 135.304 & 135.260 & -0.032 \\
\hline 25 & $2 s^{2} 2 p^{5} 3 d$ & $(1 / 2,5 / 2)_{2}$ & 0.994 & 135.522 & 135.426 & -0.071 \\
\hline 26 & $2 s^{2} 2 p^{5} 3 d$ & $(1 / 2,5 / 2)_{3}$ & 0.999 & 135.640 & 135.531 & -0.081 \\
\hline 27 & $2 s^{2} 2 p^{5} 3 d$ & $(1 / 2,3 / 2)_{1}$ & 0.971 & 136.226 & 136.065 & -0.118 \\
\hline 28 & $2 \mathrm{~s} 2 \mathrm{p}^{6} 3 \mathrm{~s}$ & $(1 / 2,1 / 2)_{1}$ & 0.994 & 139.030 & & \\
\hline 29 & $2 \mathrm{~s} 2 \mathrm{p}^{6} 3 \mathrm{~s}$ & $(1 / 2,1 / 2)_{0}$ & 0.999 & 139.808 & & \\
\hline 30 & $2 s 2 p^{6} 3 p$ & $(1 / 2,1 / 2)_{0}$ & 0.997 & 142.923 & & \\
\hline 31 & $2 s 2 p^{6} 3 p$ & $(1 / 2,1 / 2)_{1}$ & 0.990 & 143.005 & & \\
\hline 32 & $2 s^{2} p^{6} 3 p$ & $(1 / 2,3 / 2)_{2}$ & 0.997 & 143.939 & & \\
\hline 33 & $2 s^{2} p^{6} 3 p$ & $(1 / 2,3 / 2)_{1}$ & 0.991 & 144.137 & 143.835 & -0.209 \\
\hline 34 & $2 \mathrm{~s} 2 \mathrm{p}^{6} 3 \mathrm{~d}$ & $(1 / 2,3 / 2)_{1}$ & 0.999 & 148.814 & & \\
\hline 35 & $2 s 2 p^{6} 3 d$ & $(1 / 2,3 / 2)_{2}$ & 0.856 & 148.884 & & \\
\hline 36 & $2 \mathrm{~s}^{2} \mathrm{p}^{6} 3 \mathrm{~d}$ & $(1 / 2,5 / 2)_{3}$ & 0.999 & 149.027 & & \\
\hline 37 & $2 \mathrm{~s}^{2} \mathrm{p}^{6} 3 \mathrm{~d}$ & $(1 / 2,5 / 2)_{2}$ & 0.856 & 149.623 & 149.295 & -0.220 \\
\hline 38 & $2 s^{2} 2 p^{5} 4 s$ & $(3 / 2,1 / 2)_{2}$ & 1.000 & 164.361 & & \\
\hline 39 & $2 s^{2} 2 p^{5} 4 s$ & $(3 / 2,1 / 2)_{1}$ & 0.999 & 164.439 & 164.388 & -0.031 \\
\hline 40 & $2 s^{2} 2 p^{5} 4 p$ & $(3 / 2,1 / 2)_{1}$ & 0.971 & 165.929 & & \\
\hline 41 & $2 s^{2} 2 p^{5} 4 p$ & $(3 / 2,1 / 2)_{2}$ & 1.000 & 165.992 & & \\
\hline 42 & $2 s^{2} 2 p^{5} 4 p$ & $(3 / 2,1 / 2)_{3}$ & 1.000 & 166.350 & & \\
\hline 43 & $2 s^{2} 2 p^{5} 4 p$ & $(3 / 2,3 / 2)_{1}$ & 0.971 & 166.367 & & \\
\hline 44 & $2 s^{2} 2 p^{5} 4 p$ & $(3 / 2,3 / 2)_{2}$ & 1.000 & 166.469 & & \\
\hline 45 & $2 s^{2} 2 p^{5} 4 p$ & $(3 / 2,3 / 2)_{0}$ & 0.992 & 166.996 & & \\
\hline 46 & $2 s^{2} 2 p^{5} 4 d$ & $(3 / 2,3 / 2)_{0}$ & 0.997 & 168.116 & & \\
\hline 47 & $2 s^{2} 2 p^{5} 4 d$ & $(3 / 2,3 / 2)_{1}$ & 0.916 & 168.190 & 168.120 & -0.042 \\
\hline 48 & $2 s^{2} 2 p^{5} 4 d$ & $(3 / 2,3 / 2)_{3}$ & 0.999 & 168.284 & & \\
\hline 49 & $2 s^{2} 2 p^{5} 4 d$ & $(3 / 2,3 / 2)_{2}$ & 0.814 & 168.310 & & \\
\hline 50 & $2 s^{2} 2 p^{5} 4 d$ & $(3 / 2,5 / 2)_{4}$ & 1.000 & 168.314 & & \\
\hline 51 & $2 s^{2} 2 p^{5} 4 d$ & $(3 / 2,5 / 2)_{2}$ & 0.814 & 168.388 & & \\
\hline 52 & $2 s^{2} 2 p^{5} 4 d$ & $(3 / 2,5 / 2)_{3}$ & 0.999 & 168.473 & & \\
\hline 53 & $2 s^{2} 2 p^{5} 4 s$ & $(1 / 2,1 / 2)_{0}$ & 0.997 & 168.493 & & \\
\hline 54 & $2 s^{2} 2 p^{5} 4 s$ & $(1 / 2,1 / 2)_{1}$ & 0.961 & 168.510 & 168.376 & -0.080 \\
\hline 55 & $2 s^{2} 2 p^{5} 4 d$ & $(3 / 2,5 / 2)_{1}$ & 0.890 & 168.844 & 168.738 & -0.063 \\
\hline 56 & $2 s^{2} 2 p^{5} 4 f$ & $(3 / 2,5 / 2)_{1}$ & 0.999 & 169.319 & & \\
\hline 57 & $2 s^{2} 2 p^{5} 4 f$ & $(3 / 2,5 / 2)_{4}$ & 0.994 & 169.350 & & \\
\hline 58 & $2 s^{2} 2 p^{5} 4 f$ & $(3 / 2,5 / 2)_{2}$ & 0.733 & 169.359 & & \\
\hline 59 & $2 s^{2} 2 p^{5} 4 f$ & $(3 / 2,7 / 2)_{5}$ & 1.000 & 169.377 & & \\
\hline 60 & $2 s^{2} 2 p^{5} 4 f$ & $(3 / 2,7 / 2)_{3}$ & 0.884 & 169.414 & & \\
\hline 61 & $2 s^{2} 2 p^{5} 4 f$ & $(3 / 2,7 / 2)_{2}$ & 0.733 & 169.425 & & \\
\hline 62 & $2 s^{2} 2 p^{5} 4 f$ & $(3 / 2,7 / 2)_{3}$ & 0.884 & 169.434 & & \\
\hline
\end{tabular}


Table 1. (Continued.)

\begin{tabular}{|c|c|c|c|c|c|c|}
\hline No. & Conf. & Level $(\mathrm{j}-\mathrm{j})$ & Purity & $\begin{array}{l}\text { MCDF }^{\mathrm{a}} \\
\text { energy }\end{array}$ & $\begin{array}{l}\text { Exp. }{ }^{b} \\
\text { energy }\end{array}$ & $\%$ Diff. \\
\hline 63 & $2 s^{2} 2 p^{5} 4 f$ & $(3 / 2,7 / 2)_{4}$ & 0.994 & 169.460 & & \\
\hline 64 & $2 s^{2} 2 p^{5} 4 p$ & $(1 / 2,1 / 2)_{1}$ & 0.999 & 170.087 & & \\
\hline 65 & $2 s^{2} 2 p^{5} 4 p$ & $(1 / 2,3 / 2)_{1}$ & 0.999 & 170.499 & & \\
\hline 66 & $2 s^{2} 2 p^{5} 4 p$ & $(1 / 2,1 / 2)_{0}$ & 0.993 & 170.530 & & \\
\hline 67 & $2 s^{2} 2 p^{5} 4 p$ & $(1 / 2,3 / 2)_{2}$ & 1.000 & 170.534 & & \\
\hline 68 & $2 s^{2} 2 p^{5} 4 d$ & $(1 / 2,3 / 2)_{2}$ & 1.000 & 172.392 & & \\
\hline 69 & $2 s^{2} 2 p^{5} 4 d$ & $(1 / 2,5 / 2)_{2}$ & 0.999 & 172.486 & & \\
\hline 70 & $2 s^{2} 2 p^{5} 4 d$ & $(1 / 2,5 / 2)_{3}$ & 1.000 & 172.530 & & \\
\hline 71 & $2 s^{2} 2 p^{5} 4 d$ & $(1 / 2,3 / 2)_{1}$ & 0.996 & 172.690 & 172.621 & -0.040 \\
\hline 72 & $2 s^{2} 2 p^{5} 4 f$ & $(1 / 2,1 / 2)_{3}$ & 1.000 & 173.506 & & \\
\hline 73 & $2 s^{2} 2 p^{5} 4 f$ & $(1 / 2,5 / 2)_{2}$ & 1.000 & 173.531 & & \\
\hline 74 & $2 s^{2} 2 p^{5} 4 f$ & $(1 / 2,7 / 2)_{4}$ & 1.000 & 173.548 & & \\
\hline 75 & $2 s^{2} 2 p^{5} 4 f$ & $(1 / 2,7 / 2)_{3}$ & 0.999 & 173.551 & & \\
\hline 76 & $2 s 2 p^{6} 4 s$ & $(1 / 2,1 / 2)_{1}$ & 0.995 & 181.982 & & \\
\hline 77 & $2 s 2 p^{6} 4 s$ & $(1 / 2,1 / 2)_{0}$ & 0.999 & 182.268 & & \\
\hline 78 & $2 s^{2} 2 p^{5} 5 s$ & $(3 / 2,1 / 2)_{2}$ & 0.978 & 183.313 & & \\
\hline 79 & $2 s^{2} 2 p^{5} 5 s$ & $(3 / 2,1 / 2)_{1}$ & 0.933 & 183.337 & 183.258 & -0.043 \\
\hline 80 & $2 s 2 p^{6} 4 p$ & $(1 / 2,1 / 2)_{0}$ & 0.998 & 183.598 & & \\
\hline 81 & $2 s 2 p^{6} 4 p$ & $(1 / 2,1 / 2)_{1}$ & 0.925 & 183.675 & 183.420 & -0.139 \\
\hline 82 & $2 s 2 p^{6} 4 p$ & $(1 / 2,3 / 2)_{2}$ & 0.977 & 184.048 & & \\
\hline 83 & $2 \mathrm{~s} 2 \mathrm{p}^{6} 4 \mathrm{p}$ & $(1 / 2,3 / 2)_{1}$ & 0.991 & 184.082 & 183.779 & -0.165 \\
\hline 84 & $2 s^{2} 2 p^{5} 5 p$ & $(3 / 2,1 / 2)_{1}$ & 0.983 & 184.146 & & \\
\hline 85 & $2 s^{2} 2 p^{5} 5 p$ & $(3 / 2,1 / 2)_{2}$ & 0.998 & 184.155 & & \\
\hline 86 & $2 s^{2} 2 p^{5} 5 p$ & $(3 / 2,3 / 2)_{3}$ & 0.998 & 184.334 & & \\
\hline 87 & $2 s^{2} 2 p^{5} 5 p$ & $(3 / 2,3 / 2)_{1}$ & 0.984 & 184.356 & & \\
\hline 88 & $2 s^{2} 2 p^{5} 5 p$ & $(3 / 2,3 / 2)_{2}$ & 0.999 & 184.395 & & \\
\hline 89 & $2 s^{2} 2 p^{5} 5 p$ & $(3 / 2,3 / 2)_{0}$ & 0.997 & 184.662 & & \\
\hline 90 & $2 s^{2} 2 p^{5} 5 d$ & $(3 / 2,3 / 2)_{0}$ & 0.999 & 185.212 & & \\
\hline 91 & $2 s^{2} 2 p^{5} 5 d$ & $(3 / 2,3 / 2)_{1}$ & 0.930 & 185.246 & & \\
\hline 92 & $2 s^{2} 2 p^{5} 5 d$ & $(3 / 2,3 / 2)_{3}$ & 0.999 & 185.283 & & \\
\hline 93 & $2 s^{2} 2 p^{5} 5 d$ & $(3 / 2,3 / 2)_{2}$ & 0.871 & 185.299 & & \\
\hline 94 & $2 s^{2} 2 p^{5} 5 d$ & $(3 / 2,5 / 2)_{4}$ & 1.000 & 185.301 & & \\
\hline 95 & $2 s^{2} 2 p^{5} 5 d$ & $(3 / 2,5 / 2)_{2}$ & 0.871 & 185.336 & & \\
\hline 96 & $2 s^{2} 2 p^{5} 5 d$ & $(3 / 2,5 / 2)_{3}$ & 0.999 & 185.376 & & \\
\hline 97 & $2 s^{2} 2 p^{5} 5 d$ & $(3 / 2,5 / 2)$ & 0.928 & 185.554 & 185.508 & -0.025 \\
\hline 98 & $2 s^{2} 2 p^{5} 5 f$ & $(3 / 2,5 / 2)_{1}$ & 0.791 & 185.679 & & \\
\hline 99 & $2 s^{2} 2 p^{5} 5 f$ & $(3 / 2,5 / 2)_{2}$ & 0.604 & 185.723 & & \\
\hline 100 & $2 s^{2} 2 p^{5} 5 f$ & $(3 / 2,7 / 2)_{3}$ & 0.811 & 185.792 & & \\
\hline 101 & $2 s^{2} 2 p^{5} 5 f$ & $(3 / 2,5 / 2)_{4}$ & 0.996 & 185.812 & & \\
\hline 102 & $2 s^{2} 2 p^{5} 5 f$ & $(3 / 2,7 / 2)_{5}$ & 1.000 & 185.825 & & \\
\hline 103 & $2 s^{2} 2 p^{5} 5 g$ & $(3 / 2,7 / 2)_{2}$ & 0.979 & 185.839 & & \\
\hline 104 & $2 s^{2} 2 p^{5} 5 f$ & $(3 / 2,7 / 2)_{2}$ & 0.738 & 185.847 & & \\
\hline 105 & $2 s^{2} 2 p^{5} 5 f$ & $(3 / 2,5 / 2)_{3}$ & 0.936 & 185.848 & & \\
\hline 106 & $2 s^{2} 2 p^{5} 5 g$ & $(3 / 2,9 / 2)_{3}$ & 0.926 & 185.852 & & \\
\hline 107 & $2 s^{2} 2 p^{5} 5 f$ & $(3 / 2,7 / 2)_{4}$ & 0.996 & 185.866 & & \\
\hline 108 & $2 s^{2} 2 p^{5} 5 g$ & $(3 / 2,7 / 2)_{3}$ & 0.935 & 185.888 & & \\
\hline 109 & $2 s^{2} 2 p^{5} 5 g$ & $(3 / 2,7 / 2)_{5}$ & 0.994 & 185.894 & & \\
\hline 110 & $2 s^{2} 2 p^{5} 5 g$ & $(3 / 2,9 / 2)_{4}$ & 0.927 & 185.897 & & \\
\hline 111 & $2 s^{2} 2 p^{5} 5 g$ & $(3 / 2,9 / 2)_{6}$ & 1.000 & 185.904 & & \\
\hline 112 & $2 s^{2} 2 p^{5} 5 g$ & $(3 / 2,7 / 2)_{4}$ & 0.936 & 185.914 & & \\
\hline 113 & $2 s^{2} 2 p^{5} 5 g$ & $(3 / 2,9 / 2)_{5}$ & 0.994 & 185.925 & & \\
\hline 114 & $2 \mathrm{~s} 2 \mathrm{p}^{6} 4 d$ & $(1 / 2,3 / 2)_{1}$ & 0.790 & 185.980 & & \\
\hline 115 & $2 \mathrm{~s} 2 \mathrm{p}^{6} 4 \mathrm{~d}$ & $(1 / 2,3 / 2)_{2}$ & 0.763 & 185.986 & & \\
\hline 116 & $2 \mathrm{~s} 2 \mathrm{p}^{6} 4 d$ & $(1 / 2,5 / 2)_{3}$ & 0.871 & 186.021 & & \\
\hline 117 & $2 \mathrm{~s} 2 \mathrm{p}^{6} 4 \mathrm{~d}$ & $(1 / 2,5 / 2)_{2}$ & 0.877 & 186.172 & & \\
\hline 118 & $2 \mathrm{~s} 2 \mathrm{p}^{6} 4 \mathrm{f}$ & $(1 / 2,5 / 2)_{3}$ & 0.983 & 186.970 & & \\
\hline 119 & $2 \mathrm{~s} 2 \mathrm{p}^{6} 4 \mathrm{f}$ & $(1 / 2,5 / 2)_{2}$ & 0.979 & 186.994 & & \\
\hline 120 & $2 \mathrm{~s} 2 \mathrm{p}^{6} 4 \mathrm{f}$ & $(1 / 2,7 / 2)_{4}$ & 0.989 & 186.995 & & \\
\hline 121 & $2 \mathrm{~s} 2 \mathrm{p}^{6} 4 \mathrm{f}$ & $(1 / 2,7 / 2)_{3}$ & 0.976 & 187.045 & & \\
\hline 122 & $2 s^{2} 2 p^{5} 5 s$ & $(1 / 2,1 / 2)_{0}$ & 0.999 & 187.486 & & \\
\hline 123 & $2 s^{2} 2 p^{5} 5 s$ & $(1 / 2,1 / 2)_{1}$ & 0.999 & 187.504 & & \\
\hline
\end{tabular}


Table 1. (Continued.)

\begin{tabular}{lllllll}
\hline No. & Conf. & Level $(\mathrm{j}-\mathrm{j})$ & Purity & $\begin{array}{l}\text { MCDF } \\
\text { energy }\end{array}$ & $\begin{array}{l}\text { Exp. }^{\mathrm{b}} \\
\text { energy }\end{array}$ & \% Diff. \\
\hline 124 & $2 \mathrm{~s}^{2} 2 \mathrm{p}^{5} 5 \mathrm{p}$ & $(1 / 2,1 / 2)_{1}$ & 0.999 & 188.283 & & \\
125 & $2 \mathrm{~s}^{2} 2 \mathrm{p}^{5} 5 \mathrm{p}$ & $(1 / 2,1 / 2)_{0}$ & 0.998 & 188.475 & \\
126 & $2 \mathrm{~s}^{2} 2 \mathrm{p}^{5} 5 \mathrm{p}$ & $(1 / 2,3 / 2)_{1}$ & 1.000 & 188.489 & \\
127 & $2 \mathrm{~s}^{2} 2 \mathrm{p}^{5} 5 \mathrm{p}$ & $(1 / 2,3 / 2)_{2}$ & 0.999 & 188.505 & \\
128 & $2 \mathrm{~s}^{2} 2 \mathrm{p}^{5} 5 \mathrm{~d}$ & $(1 / 2,3 / 2)_{2}$ & 1.000 & 189.406 & \\
129 & $2 \mathrm{~s}^{2} 2 \mathrm{p}^{5} 5 \mathrm{~d}$ & $(1 / 2,5 / 2)_{2}$ & 1.000 & 189.457 & \\
130 & $2 \mathrm{~s}^{2} 2 \mathrm{p}^{5} 5 \mathrm{~d}$ & $(1 / 2,5 / 2)_{3}$ & 1.000 & 189.476 & \\
131 & $2 \mathrm{~s}^{2} 2 \mathrm{p}^{5} 5 \mathrm{~d}$ & $(1 / 2,3 / 2)_{1}$ & 0.999 & 189.547 & 189.492 & -0.020 \\
132 & $2 \mathrm{~s}^{2} 2 \mathrm{p}^{5} 5 \mathrm{f}$ & $(1 / 2,5 / 2)_{3}$ & 0.999 & 189.957 & \\
133 & $2 \mathrm{~s}^{2} 2 \mathrm{p}^{5} 5 \mathrm{f}$ & $(1 / 2,5 / 2)_{2}$ & 1.000 & 189.974 & \\
134 & $2 \mathrm{~s}^{2} 2 \mathrm{p}^{5} 5 \mathrm{f}$ & $(1 / 2,7 / 2)_{4}$ & 1.000 & 189.980 & \\
135 & $2 \mathrm{~s}^{2} 2 \mathrm{p}^{5} 5 \mathrm{f}$ & $(1 / 2,7 / 2)_{3}$ & 0.999 & 189.982 & \\
136 & $2 \mathrm{~s}^{2} 2 \mathrm{p}^{5} 5 \mathrm{~g}$ & $(1 / 2,7 / 2)_{4}$ & 0.999 & 190.039 & \\
137 & $2 \mathrm{~s}^{2} 2 \mathrm{p}^{5} 5 \mathrm{~g}$ & $(1 / 2,7 / 2)_{3}$ & 0.998 & 190.049 & \\
138 & $2 \mathrm{~s}^{2} 2 \mathrm{p}^{5} 5 \mathrm{~g}$ & $(1 / 2,9 / 2)_{5}$ & 1.000 & 190.051 & \\
139 & $2 \mathrm{~s}^{2} 2 \mathrm{p}^{5} 5 \mathrm{~g}$ & $(1 / 2,9 / 2)_{4}$ & 0.997 & 190.060 & \\
\hline
\end{tabular}

${ }^{a}$ Present multi-configuration Dirac-Fock calculation.

${ }^{\mathrm{b}}$ NIST Atomic Spectroscopic Data:

http://physics.nist.gov/PhysRefData/contents-atomic.html.

Table 2. Weighted length oscillator strengths and ratios of velocity to length oscillator strengths for all electric-dipole transitions to the ground state in $\mathrm{Kr}^{26+}$.

\begin{tabular}{rllll}
\hline $\begin{array}{l}\text { Level } \\
\text { no. }\end{array}$ & Designation & Present gf & $\begin{array}{l}\text { vel/ } \\
\text { len }\end{array}$ & gf (Rice et al) \\
\hline 3 & $2 \mathrm{~s}^{2} 2 \mathrm{p}^{5} 3 \mathrm{~s}(3 / 2,1 / 2)_{1}$ & $1.34 \times 10^{-1}$ & 0.98 & - \\
7 & $2 \mathrm{~s}^{2} 2 \mathrm{p}^{5} 3 \mathrm{~s}(1 / 2,1 / 2)_{1}$ & $8.45 \times 10^{-2}$ & 0.98 & - \\
17 & $2 \mathrm{~s}^{2} 2 \mathrm{p}^{5} 3 \mathrm{~d}(3 / 2,3 / 2)_{1}$ & $6.41 \times 10^{-3}$ & 0.95 & $7.79 \times 10^{-3}$ \\
23 & $2 \mathrm{~s}^{2} 2 \mathrm{p}^{5} 3 \mathrm{~d}(3 / 2,5 / 2)_{1}$ & $1.55 \times 10^{0}$ & 0.96 & $1.54 \times 10^{0}$ \\
27 & $2 \mathrm{~s}^{2} 2 \mathrm{p}^{5} 3 \mathrm{~d}(1 / 2,3 / 2)_{1}$ & $1.93 \times 10^{0}$ & 0.96 & $1.94 \times 10^{0}$ \\
31 & $2 \mathrm{~s}^{6} \mathrm{p}^{6} 3 \mathrm{p}(1 / 2,1 / 2)_{1}$ & $8.97 \times 10^{-2}$ & 1.00 & $8.75 \times 10^{-2}$ \\
33 & $2 \mathrm{~s}^{6} \mathrm{p}^{6} 3 \mathrm{p}(1 / 2,3 / 2)_{1}$ & $3.05 \times 10^{-1}$ & 1.00 & $3.05 \times 10^{-1}$ \\
39 & $2 \mathrm{~s}^{2} 2 \mathrm{p}^{5} 4 \mathrm{~s}(3 / 2,1 / 2)_{1}$ & $2.48 \times 10^{-2}$ & 0.91 & $2.47 \times 10^{-2}$ \\
47 & $2 \mathrm{~s}^{2} 2 \mathrm{p}^{5} 4 \mathrm{~d}(3 / 2,3 / 2)_{1}$ & $3.26 \times 10^{-3}$ & 0.93 & - \\
54 & $2 \mathrm{~s}^{2} 2 \mathrm{p}^{5} 4 \mathrm{~s}(1 / 2,1 / 2)_{1}$ & $7.94 \times 10^{-2}$ & 0.93 & $6.80 \times 10^{-2}$ \\
55 & $2 \mathrm{~s}^{2} 2 \mathrm{p}^{5} 4 \mathrm{~d}(3 / 2,5 / 2)_{1}$ & $4.29 \times 10^{-1}$ & 0.94 & $4.34 \times 10^{-1}$ \\
71 & $2 \mathrm{~s}^{2} 2 \mathrm{p}^{5} 4 \mathrm{~d}(1 / 2,3 / 2)_{1}$ & $3.38 \times 10^{-1}$ & 0.94 & $3.30 \times 10^{-1}$ \\
79 & $2 \mathrm{~s}^{2} 2 \mathrm{p}^{5} 5 \mathrm{~s}(3 / 2,1 / 2)_{1}$ & $2.97 \times 10^{-2}$ & 0.82 & $3.33 \times 10^{-2}$ \\
81 & $2 \mathrm{~s}^{6} \mathrm{p}^{6} 4 \mathrm{p}(1 / 2,1 / 2)_{1}$ & $1.77 \times 10^{-2}$ & 1.00 & $1.30 \times 10^{-2}$ \\
83 & $2 \mathrm{~s}^{6} \mathrm{p}^{6} 4 \mathrm{p}(1 / 2,3 / 2)_{1}$ & $1.20 \times 10^{-1}$ & 0.95 & $1.17 \times 10^{-1}$ \\
91 & $2 \mathrm{~s}^{2} 2 \mathrm{p}^{5} 5 \mathrm{~d}(3 / 2,3 / 2)_{1}$ & $4.98 \times 10^{-4}$ & 0.90 & - \\
97 & $2 \mathrm{~s}^{2} 2 \mathrm{p}^{5} 5 \mathrm{~d}(3 / 2,5 / 2)_{1}$ & $2.24 \times 10^{-1}$ & 0.91 & $2.33 \times 10^{-1}$ \\
123 & $2 \mathrm{~s}^{2} 2 \mathrm{p}^{5} 5 \mathrm{~s}(1 / 2,1 / 2)_{1}$ & $2.50 \times 10^{-3}$ & 0.62 & - \\
131 & $2 \mathrm{~s}^{2} 2 \mathrm{p}^{5} 5 \mathrm{~d}(1 / 2,3 / 2)_{1}$ & $1.31 \times 10^{-1}$ & 0.91 & $1.37 \times 10^{-1}$ \\
\hline
\end{tabular}

suite of programs [23-25]. In order to combine these two sets of programs, the Hamiltonian matrix elements generated by the inner-region DARC codes for a given $J \Pi$ symmetry were reformatted to be consistent with those in the BP suite of codes $[26,27]$. They may be run with or without radiation damping. A flow diagram for calculations that include damping is shown in a paper on electron-impact excitation of $\mathrm{W}^{46+}$ [20]. The CC expansion for the scattering calculations included all 139 levels listed in table 1 . The size of the $R$-matrix 'box' was 3.6 au and we employed 33 basis orbitals to represent the $(N+1)$-electron continuum per angular momentum. All partial waves from $J=1 / 2$ to $71 / 2$ were included explicitly and contributions from higher partial waves were estimated using a top-up procedure [28].

Below the highest excitation threshold, a very fine energy mesh must be implemented in order to resolve the majority of narrow resonances. For this reason, for all partial waves from $J=1 / 2$ to $21 / 2$, we first performed a calculation with 80000 points in the energy range from the first threshold to just above the highest threshold. We then doubled the number of mesh points to 160000 . By comparing effective collision strengths from these two calculations, we were able to confirm that most resonances were resolved. Our final 160000 points calculation employed an energy-mesh spacing of $4.36 \times$ $10^{-4}$ Ry. An additional 200 energy mesh points for these lower partial waves were used to span the energy range from just above the highest threshold to a maximum energy of $570 \mathrm{Ry}$. For the higher partial waves from $J=23 / 2$ to $71 / 2$, we calculated 2000 mesh points to cover the energy range from the first threshold to 570 Ry.

In table 4, we compare cross sections from the present calculation with those from the earlier 27-level BP $R$-matrix calculation of Gupta et al [13] and the relativistic DW calculation of Reed [11]. The cross sections from these two prior calculations were determined from collision strengths that are given at energies of $150 \mathrm{Ry}, 300 \mathrm{Ry}$ and $500 \mathrm{Ry}$ in [13]. Since no resonances are included in the relativistic DW calculation and only those resonant states attached to the $2 \mathrm{p}^{5} 3 \mathrm{l}$ configurations are included in the BP $R$-matrix calculation, such comparisons are not meaningful for energies below the highest excitation threshold in the present calculation. Thus, only the values at $300 \mathrm{Ry}$ and $500 \mathrm{Ry}$ were employed here. The cross sections from the present calculation are higher than those from either the BP $R$-matrix or the relativistic DW calculations for the vast majority of transitions, especially at an energy of 500 Ry. At 300 Ry, the average difference between the present results and those of Gupta et al [13] is $8.5 \%$, while the average difference between the present results and those of 
Table 3. Diagnostic radiative transitions to the ground level in $\mathrm{Kr}^{26+}$ with theoretical and experimental wavelengths and theoretical radiative rates.

\begin{tabular}{llllll}
\hline Upper level & Transition & Label & Th. $\lambda(\AA)$ & Exp. $\lambda(\AA)$ & MCDF A $\left(\mathrm{s}^{-1}\right)$ \\
\hline $2 \mathrm{p}^{5} 3 \mathrm{~s}(3 / 2,1 / 2)_{2}$ & $(2 \rightarrow 1)$ & M2 & 7.5191 & 7.5184 & $5.40 \times 10^{6}$ \\
$2 \mathrm{p}^{5} 3 \mathrm{~s}(3 / 2,1 / 2)_{1}$ & $(3 \rightarrow 1)$ & 3G & 7.5047 & 7.5038 & $5.27 \times 10^{12}$ \\
$2 \mathrm{p}^{5} 3 \mathrm{~s}(1 / 2,1 / 2)_{1}$ & $(7 \rightarrow 1)$ & $3 \mathrm{~F}$ & 7.2651 & 7.2669 & $3.56 \times 10^{12}$ \\
$2 \mathrm{p}^{5} 3 \mathrm{~d}(3 / 2,3 / 2)_{1}$ & $(17 \rightarrow 1)$ & 3E & 6.9596 & 6.9591 & $2.94 \times 10^{11}$ \\
$2 \mathrm{p}^{5} 3 \mathrm{~d}(3 / 2,5 / 2)_{1}$ & $(23 \rightarrow 1)$ & 3D & 6.8748 & 6.8787 & $7.30 \times 10^{13}$ \\
$2 \mathrm{p}^{5} 3 \mathrm{~d}(1 / 2,3 / 2)_{1}$ & $(27 \rightarrow 1)$ & 3C & 6.6893 & 6.6973 & $9.60 \times 10^{13}$ \\
\hline
\end{tabular}

Table 4. Cross sections in units of $\mathrm{cm}^{2}$ for excitations from the $2 \mathrm{p}^{6}{ }^{1} \mathrm{~S}_{0}$ ground level to the first 26 excited levels in $\mathrm{Kr}^{26+}$ at excitation energies of $300 \mathrm{Ry}$ and $500 \mathrm{Ry}$. The numbers in parentheses denote powers of 10 .

\begin{tabular}{|c|c|c|c|c|c|c|}
\hline \multirow[b]{2}{*}{ Upper level } & \multicolumn{3}{|c|}{$300 \mathrm{Ry}$} & \multicolumn{3}{|c|}{$500 \mathrm{Ry}$} \\
\hline & $\mathrm{PRM}^{\mathrm{a}}$ & Rel DW ${ }^{b}$ & $\operatorname{res} \mathrm{RM}^{\mathrm{c}}$ & $\mathrm{PRM}^{\mathrm{a}}$ & el DW ${ }^{b}$ & Pres $\mathrm{RM}^{\mathrm{c}}$ \\
\hline $53 \mathrm{~s}$ & $x_{1}$ & $80(-23)$ & or & $08(-$ & $05(-23)$ & $73(-23)$ \\
\hline $1 / 2)_{1}$ & $22(-22)$ & $6.63(-22)$ & $29(-22)$ & $87(-22)$ & $.43(-22)$ & $.04(-22)$ \\
\hline$p^{5} 3 p(3 / 2,1 / 2)_{1}$ & $.60(-22)$ & $1.73(-22)$ & $.85(-22)$ & $87(-23)$ & $4.11(-23)$ & $5.26(-23)$ \\
\hline$p^{5} 3 p(3 / 2,1 / 2)_{2}$ & $4.25(-22)$ & $4.72(-22)$ & $4.88(-22)$ & $.92(-22)$ & $2.52(-22)$ & $3.14(-22)$ \\
\hline$p^{5} 3 s(1 / 2,1 / 2)_{0}$ & $1.90(-23)$ & $1.76(-23)$ & $2.01(-2$ & $37(-24)$ & $4.40(-24)$ & $5.53(-24)$ \\
\hline $53 \mathrm{~s}(1 / 2,1 / 2)_{1}$ & $2.99(-22)$ & $4.16(-22)$ & $52(-7-2-3$ & $60(-22)$ & $3.18(-22)$ & $.24(-22)$ \\
\hline $53 \mathrm{p}(3 /$ & $30(-22)$ & $2.20(-22)$ & 346 & 23 & 184 & \\
\hline $\mathrm{p}^{5} 3 \mathrm{p}$ & & $1.06(-$ & & & & \\
\hline $\mathrm{p}^{5} 3$ & & 3.9 & & & & \\
\hline 53 & & & & & & $(-21)$ \\
\hline & & 9.05 & & & & $2.69(-23)$ \\
\hline & & 1.1 & & & & $3.30(-23)$ \\
\hline & & & & & & $4(-22)$ \\
\hline 2) & & & & & & $2.58(-23)$ \\
\hline $1 / 2,1 / 2)_{0}$ & $5.48(-$ & & & & & $3.27(-21)$ \\
\hline $\mathrm{p}^{5} 3 \mathrm{~d}(3 / 2,3 / 2)_{1}$ & $3.08(-22)$ & & & & & $1.20(-22)$ \\
\hline $2 p^{5} 3 d(3 / 2,3 / 2)_{3}$ & $4.75(-22)$ & $4.66(-22)$ & $4.78(-$ & $2.39(-$ & $2.26(-$ & $2.76(-22)$ \\
\hline $2 p^{5} 3 d(3 / 2,5 / 2)_{2}$ & $2.92(-2$ & $2.90(-2$ & $3.00(-$ & $.86(-$ & $5.72(-$ & $7.17(-23)$ \\
\hline $2 p^{5} 3 d(3 / 2,5 / 2)_{4}$ & $3.11(-2$ & $2.99(-22)$ & $3.17(-$ & $.00(-$ & $5.72(-$ & $7.28(-23)$ \\
\hline $2 \mathrm{p}^{5} 3 \mathrm{~d}(3 / 2,3 / 2)_{2}$ & $1.33(-2)$ & $1.26(-22)$ & $1.42(-2$ & $2.42(-23)$ & $2.20(-$ & $3.15(-23)$ \\
\hline $2 p^{5} 3 d(3 / 2,5 / 2)_{3}$ & $3.67(-2$ & $3.61(-2$ & $3.62(-$ & $2.02(-$ & $1.88(-$ & $2.29(-22)$ \\
\hline$n^{5} 3 d(3 / 25 / 2)$ & $1.30(-$ & $1.46(-$ & $1.45(-$ & $9.74(-$ & $9.93(-$ & $1.20(-20)$ \\
\hline $3 / 2)$ & $1.55(-$ & $1.47(-$ & $1.58(-$ & $2.89(-$ & $2.79(-$ & $3.58(-23)$ \\
\hline 532 & $2.44(-2$ & $2.32(-22)$ & $2.44(-$ & $4.77(-2$ & $4.55(-$ & $5.74(-23)$ \\
\hline $2 p^{5} 3 d$ & & $4.25(-22)$ & & & & $2.58(-22)$ \\
\hline $2 p^{5} 3 d(1 / 2,3 / 2)_{1}$ & $1.64(-20)$ & $1.46(-20)$ & $1.72(-20)$ & $1.21(-20)$ & $1.19(-20)$ & $1.40(-20)$ \\
\hline
\end{tabular}

\footnotetext{
${ }^{\text {a }}$ Determined from the 27-level BP $R$-matrix collisions strengths of Gupta et al [13].

${ }^{\mathrm{b}}$ Determined from the relativistic DW collisions strengths of Reed [11] given in [13].

${ }^{\text {c }}$ From the present 139 -level Dirac $R$-matrix calculation.
}

Reed [11] is $6.2 \%$. At 500 Ry, these average differences have increased to $20.8 \%$ and $23.8 \%$, respectively.

Some of these differences are due to the different target states used in each calculation. For example, the energies in the present calculation are in better agreement with experiment than those from the BP structure calculations of Gupta et al [13]. However, the fact that the present cross sections are in general higher, and these differences increase substantially with energy strongly suggests that they are due primarily to a lack of completeness in the partial-wave sums used in the earlier calculations. The sum used by Gupta et al [13] only included partial waves up through $J=17 / 2$; they then topped up their dipole-allowed transitions using a procedure similar to ours. This expansion is not sufficiently complete to achieve convergence, especially at energies as high as $500 \mathrm{Ry}$. A description of the partial-wave sum employed in the relativistic DW calculation is not available.

In figure 1, we show excitation cross sections from the ground level to the four levels arising from the $2 s^{2} 2 p^{5} 3 s$ configuration. The dotted curves with the complex resonance structures are from the calculation with damping. In order to show the effects of radiation damping on these cross sections, we also calculated the excitation cross sections for these four transitions convoluted with a $2.94 \mathrm{Ry}(40 \mathrm{eV})$ Gaussian and compared the results with (solid curves) and without (the long dashed curves) damping. As can be seen, the cross sections are quite close and a calculation without damping would be sufficiently accurate, at least for these selected excitations.

The most pronounced feature for all four of these cross sections is the very large contributions from resonances 


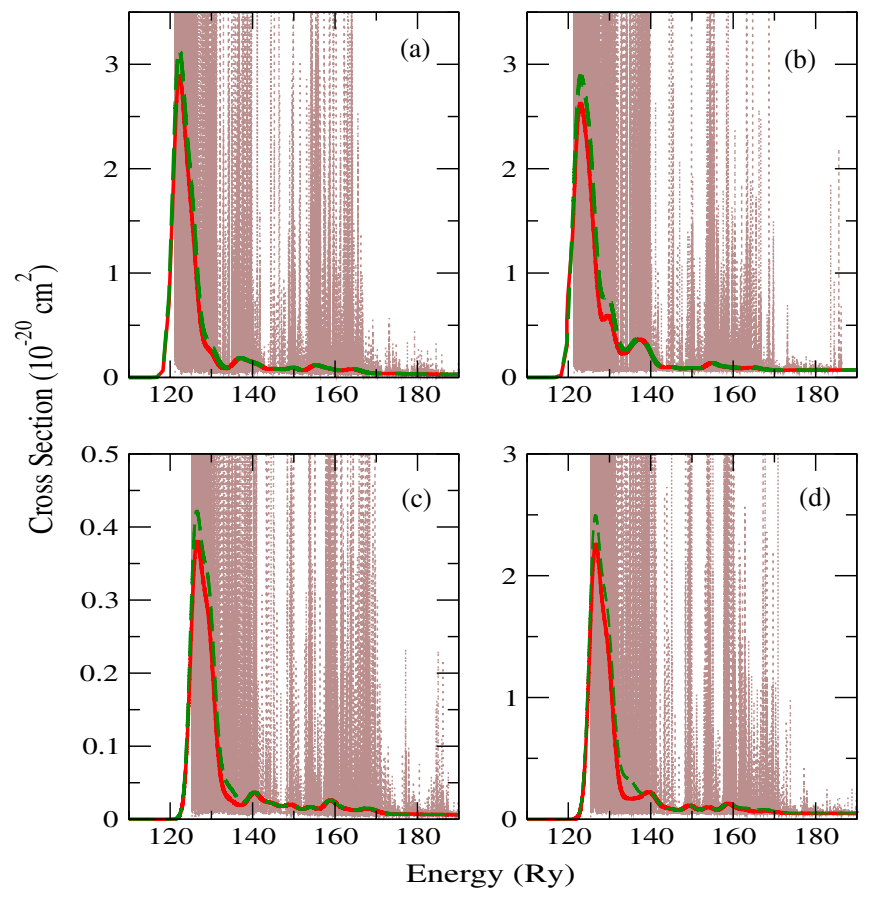

Figure 1. Electron-impact excitation cross sections for transitions from the $2 s^{2} 2 \mathrm{p}^{6}{ }^{1} \mathrm{~S}_{0}$ ground level to the levels of the $2 \mathrm{~s}^{2} 2 \mathrm{p}^{5} 3 \mathrm{~s}$ configuration: (a) to $(3 / 2,1 / 2)_{2}$ (level 2); (b) to $(3 / 2,1 / 2)_{1}$ (level 3); (c) to $(1 / 2,1 / 2)_{0}$ (level 6) and (d) to $(1 / 2,1 / 2)_{1}$ (level 7). The dotted curves with the pronounced resonance structures are from the 139-level $R$-matrix calculation with radiation damping; the smooth solid curves are the cross sections from the 139-level calculation with damping convoluted with a 2.94 Ry Gausssian; and the long dashed curves are the cross sections from the 139-level calculation with no radiative damping convoluted with a 2.94 Ry Gausssian.

at low energy. Clearly, a DW calculation that does not include perturbative calculations of resonant recombination followed by autoionization would be completely inaccurate. Furthermore, it is clear that the resonant contributions above the highest $2 \mathrm{p}^{5} 3 \mathrm{~d}$ level at approximately $136 \mathrm{Ry}$ are smaller than those at lower energy; however, they are far from negligible. In addition, some of the contributions below this energy arise from resonances attached to levels above the highest $2 \mathrm{p}^{5} 3 \mathrm{~d}$ level. Thus the 27 -level BP $R$-matrix results of Gupta et al [13] will have noticeably smaller resonance contributions for excitation to the $2 \mathrm{p}^{5} 3 \mathrm{~s}$ levels than the cross sections shown here.

In figure 2, we show similar curves for excitation from the ground level to four of the twelve levels arising from the $2 s^{2} 2 p^{5} 3 d$ configuration. By comparing the convoluted cross sections, we see that the effects of radiation damping are again very small. Furthermore, the resonance contributions for the non-dipole excitation to the $2 s^{2} 2 p^{5} 3 d(3 / 2,3 / 2)_{0}$ level and the weak dipole excitation to the $2 s^{2} 2 p^{5} 3 d(3 / 2,3 / 2)_{1}$ level are quite important and they are dominated by resonances attached to levels above the highest level of the $2 s^{2} 2 p^{5} 3 d$ configuration. However, as one would expect, the effects of resonances on the cross sections for the strong dipole excitations to the $2 \mathrm{~s}^{2} 2 \mathrm{p}^{5} 3 \mathrm{~d}$ $(3 / 2,5 / 2)_{1}$ and the $2 s^{2} 2 p^{5} 3 d(1 / 2,3 / 2)_{1}$ levels are far less important.

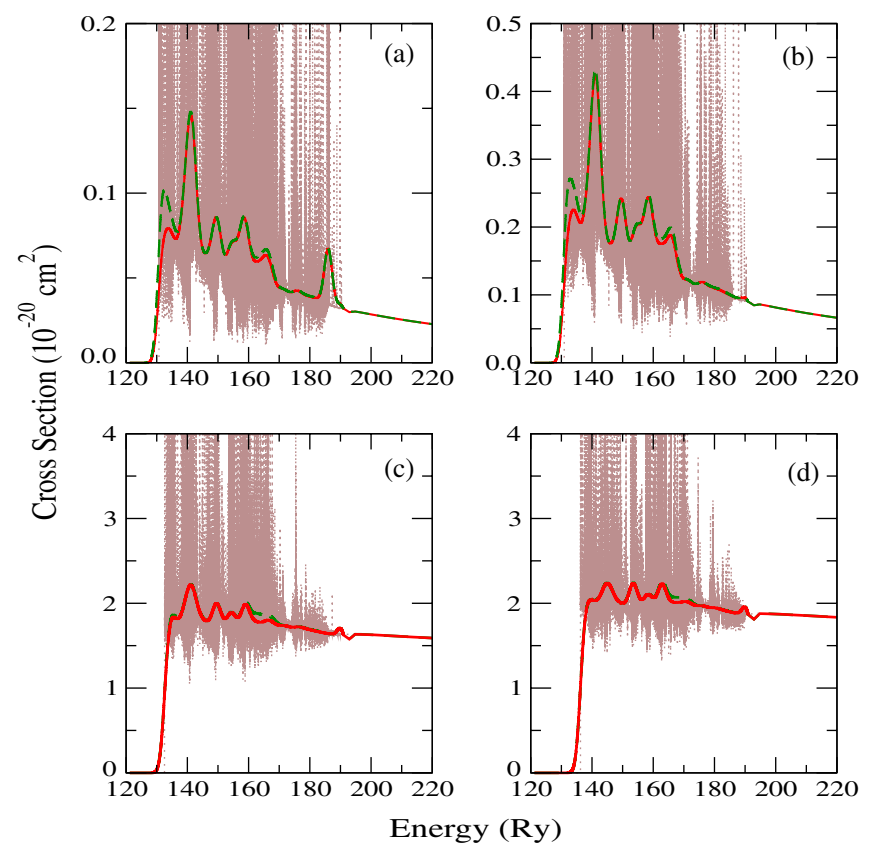

Figure 2. Electron-impact excitation cross sections for transitions from the $2 \mathrm{~s}^{2} 2 \mathrm{p}^{6}{ }^{1} \mathrm{~S}_{0}$ ground level to four levels of the $2 \mathrm{~s}^{2} 2 \mathrm{p}^{5} 3 \mathrm{~d}$ configuration: (a) to $(3 / 2,3 / 2)_{0}$ (level 15$)$; (b) to $(3 / 2,3 / 2)_{1}$ (level 17); (c) to $(3 / 2,5 / 2)_{1}$ (level 23); (d) to $(1 / 2,3 / 2)_{1}$ (level 27). The dotted curves with the pronounced resonance structures are from the 139-level $R$-matrix calculation with radiation damping; the smooth solid curves are the cross sections from the 139-level calculation with damping convoluted with a 2.94 Ry Gausssian; and the long dashed curves are the cross sections from the 139-level calculation with no radiative damping convoluted with a 2.94 Ry Gausssian.

In table 5, we present effective collision strengths calculated both with and without damping for excitation from the ground level to the first 26 excited levels over six temperatures from $5 \times 10^{6} \mathrm{~K}$ to $3 \times 10^{7} \mathrm{~K}$. As can be seen, the effects of damping are again relatively small for these 26 transitions with only three exceeding $10 \%$. When averaged over all 9591 transitions between the 139 levels and over ten temperatures from $5 \times 10^{6} \mathrm{~K}$ to $5 \times 10^{7} \mathrm{~K}$, the average difference between the effective collision strengths with and without damping was only $1.58 \%$. The effective collision strengths, with radiation damping, along with electric-dipole, electric-quadrupole and magnetic-dipole radiative rates are now available at the Oak Ridge National Laboratory (ORNL) Controlled Fusion Atomic Data Center (CFADC) website, at http://www-cfadc.phy.ornl.gov/data_and_codes/home.html.

Finally, we have performed collisional-radiative modeling calculations that utilize the present radiative rates and collisional rates with radiation damping to calculate intensity line ratios involving the six transitions given in table 3 . If one assumes that the main population mechanism of any excited level is direct excitation from the ground level $(g)$, then the intensity line ratio for the transition $i \rightarrow g$ to the transition $j \rightarrow g$ is given by

$$
\frac{I_{i \rightarrow g}}{I_{j \rightarrow g}}=\frac{Q_{g \rightarrow i}}{Q_{g \rightarrow j}} \frac{B_{i \rightarrow g}}{B_{j \rightarrow g}} .
$$


Table 5. Effective collision strengths for excitations from the $2 \mathrm{p}^{6}{ }^{1} \mathrm{~S}_{0}$ ground level to the first 26 excited levels in $\mathrm{Kr}^{26+}$. For each transition, the first row is from the $R$-matrix calculation without damping while the second row is from the calculation with damping. The last column lists the percentage differences between these two calculations averaged over the six temperatures. The numbers in parentheses denote powers of 10 .

\begin{tabular}{|c|c|c|c|c|c|c|c|}
\hline \multirow[b]{2}{*}{ Upper level } & \multicolumn{7}{|c|}{ Electron temperature $(\mathrm{K})$} \\
\hline & $5 \times 10^{6}$ & $7 \times 10^{6}$ & $9 \times 10^{6}$ & $1 \times 10^{7}$ & $2 \times 10^{7}$ & $3 \times 10^{7}$ & $\begin{array}{l}\text { Avg. \% } \\
\text { diff. }\end{array}$ \\
\hline \multirow[t]{2}{*}{$2 \mathrm{p}^{5} 3 \mathrm{~s}(3 / 2,1 / 2)_{2}$} & $8.29(-3)$ & $6.28(-3)$ & $5.09(-3)$ & $4.66(-3)$ & $2.61(-3)$ & $1.85(-3)$ & \\
\hline & $7.44(-3)$ & $5.65(-3)$ & $4.60(-3)$ & $4.21(-3)$ & $2.37(-3)$ & $1.69(-3)$ & 6.0 \\
\hline \multirow[t]{2}{*}{$2 \mathrm{p}^{5} 3 \mathrm{~s}(3 / 2,1 / 2)_{1}$} & $9.36(-3)$ & $7.39(-3)$ & $6.27(-3)$ & $5.88(-3)$ & $4.28(-3)$ & $4.00(-3)$ & \\
\hline & $8.14(-3)$ & $6.48(-3)$ & $5.54(-3)$ & $5.21(-3)$ & $3.94(-3)$ & $3.76(-3)$ & 7.4 \\
\hline \multirow[t]{2}{*}{$2 \mathrm{p}^{5} 3 \mathrm{p}(3 / 2,1 / 2)_{1}$} & $3.49(-3)$ & $2.91(-3)$ & $2.54(-3)$ & $2.39(-3)$ & $1.61(-3)$ & $1.26(-3)$ & \\
\hline & $3.03(-3)$ & $2.58(-3)$ & $2.27(-3)$ & $2.15(-3)$ & $1.48(-3)$ & $1.17(-3)$ & 6.9 \\
\hline \multirow[t]{2}{*}{$2 \mathrm{p}^{5} 3 \mathrm{p}(3 / 2,1 / 2)_{2}$} & $4.30(-3)$ & $3.69(-3)$ & $3.31(-3)$ & $3.17(-3)$ & $2.50(-3)$ & $2.26(-3)$ & \\
\hline & $3.85(-3)$ & $3.36(-3)$ & $3.05(-3)$ & $2.93(-3)$ & $2.37(-3)$ & $2.17(-3)$ & 5.2 \\
\hline \multirow[t]{2}{*}{$2 \mathrm{p}^{5} 3 \mathrm{~s}(1 / 2,1 / 2)_{0}$} & $1.32(-3)$ & $1.01(-3)$ & $8.27(-4)$ & $7.60(-4)$ & $4.33(-4)$ & $3.11(-4)$ & \\
\hline & $1.14(-3)$ & $8.80(-4)$ & $7.22(-4)$ & $6.65(-4)$ & $3.84(-4)$ & $2.78(-4)$ & 7.9 \\
\hline \multirow{2}{*}{$2 \mathrm{p}^{5} 3 \mathrm{~s}(1 / 2,1 / 2)_{1}$} & $7.69(-3)$ & $5.99(-3)$ & $5.02(-3)$ & $4.68(-3)$ & $3.20(-3)$ & $2.84(-3)$ & \\
\hline & $6.54(-$ & 5.14 & $4.33(-3)$ & $(-3)$ & $2.88(-3)$ & $2.62(-3)$ & 8.7 \\
\hline \multirow[t]{2}{*}{$2 p^{5} 3 p(3 / 2,3 / 2)_{3}$} & $4.03(-3)$ & $3.45(-3)$ & $3.05(-3)$ & $2.89(-3)$ & $1.98(-3)$ & $1.56(-3)$ & \\
\hline & $3.85(-3)$ & $3.31(-3)$ & $2.94(-3)$ & $2.79(-3)$ & $1.93(-3)$ & $1.52(-3)$ & 2.3 \\
\hline \multirow[t]{2}{*}{$2 p^{5} 3 p(3 / 2,3 / 2)_{1}$} & $3.14(-3)$ & $2.58(-3)$ & $2.21(-3)$ & $2.07(-3)$ & $1.33(-3)$ & $1.01(-3)$ & \\
\hline & $2.20(-3)$ & $1.88(-3)$ & $1.66(-3)$ & $1.57(-3)$ & $1.07(-3)$ & $8.37(-4)$ & 17.5 \\
\hline \multirow{2}{*}{$2 p^{5} 3 p(3 / 2,3 / 2)_{2}$} & $3.36(-3)$ & $2.94(-3)$ & $2.67(-3)$ & $2.57(-3)$ & $2.07(-3)$ & $1.90(-3)$ & \\
\hline & $3.12(-3)$ & 2.76 & $2.53(-3)$ & $2.44(-3)$ & $2.00(-3)$ & $1.85(-3)$ & 3.5 \\
\hline \multirow{2}{*}{$2 \mathrm{p}^{5} 3 \mathrm{p}(3 / 2,3 / 2)_{0}$} & $8.67(-3)$ & $8.19(-3)$ & $7.90(-3)$ & $7.80(-3)$ & $7.36(-3)$ & $7.26(-3)$ & \\
\hline & $7.52(-3)$ & $7.34(-3)$ & $7.23(-3)$ & $(-3)$ & $7.04(-3)$ & $7.05(-3)$ & 6.0 \\
\hline \multirow{2}{*}{$2 p^{5} 3 p(1 / 2,1 / 2)_{1}$} & $2.69(-3)$ & $2.18(-3)$ & $1.86(-3)$ & $1.74(-3)$ & $1.11(-3)$ & $8.41(-4)$ & \\
\hline & $1.76(-3)$ & $1.50(-3)$ & $1.32(-3)$ & $(-3)$ & $8.54(-4)$ & $6.71(-4)$ & 20.8 \\
\hline \multirow[t]{2}{*}{$2 p^{5} 3 p(1 / 2,3 / 2)_{1}$} & $2.80(-3)$ & 2.33 & $2.02(-3)$ & $1.90(-3)$ & $1.25(-3)$ & $9.57(-4)$ & \\
\hline & $2.20(-3)$ & 1.90 & $1.68(-3)$ & $1.59(-3)$ & $1.08(-3)$ & $8.47(-4)$ & 11.5 \\
\hline \multirow[t]{2}{*}{$2 p^{5} 3 p(1 / 2,3 / 2)_{2}$} & $3.27(-3)$ & $2.92(-3)$ & $2.68(-3)$ & $2.60(-3)$ & $2.13(-3)$ & $1.96(-3)$ & \\
\hline & $2.90(-3)$ & $2.64(-3)$ & $2.46(-3)$ & $2.39(-3)$ & $2.03(-3)$ & $1.89(-3)$ & 5.6 \\
\hline \multirow[t]{2}{*}{$2 \mathrm{p}^{5} 3 \mathrm{~d}(3 / 2,3 / 2)_{0}$} & $1.24(-3)$ & $1.12(-3)$ & $1.02(-3)$ & $9.80(-4)$ & $7.19(-4)$ & $5.82(-4)$ & \\
\hline & $1.17(-3)$ & $1.06(-3)$ & $9.79(-4)$ & $(-4)$ & $6.99(-4)$ & $5.68(-4)$ & 2.8 \\
\hline \multirow[t]{2}{*}{$2 \mathrm{p}^{5} 3 \mathrm{p}(1 / 2,1 / 2)_{0}$} & $1.71(-2)$ & $1.69(-2)$ & $1.69(-2)$ & $1.69(-2)$ & $1.70(-2)$ & $1.73(-2)$ & \\
\hline & $1.61(-2)$ & $1.62(-2)$ & $1.63(-2)$ & $(-2)$ & $1.68(-2)$ & $1.71(-2)$ & 2.5 \\
\hline \multirow[t]{2}{*}{$2 p^{5} 3 d(3 / 2,3 / 2)_{1}$} & $3.45(-3)$ & 3.12 & $2.86(-3)$ & $-3)$ & $2.08(-3)$ & $1.74(-3)$ & \\
\hline & $3.28(-3)$ & $2.99(-3)$ & $2.75(-3)$ & $-3)$ & 2.03 & $1.70(-3)$ & 2.4 \\
\hline $2 p^{5} 3 d(3 / 2,3 / 2)_{3}$ & $3.80(-3)$ & $3.41(-3)$ & $3.14(-3)$ & $-3)$ & $2.44(-3)$ & $2.19(-3)$ & \\
\hline & $3.46(-3)$ & $3.15(-3)$ & $2.93(-3)$ & $(-3)$ & $2.34(-3)$ & $2.12(-3)$ & 4.4 \\
\hline $2 p^{5} 3 d(3 / 2,5 / 2)_{2}$ & $4.13(-3)$ & $3.67(-3)$ & $3.32(-3)$ & $-3)$ & $2.27(-3)$ & $1.81(-3)$ & \\
\hline & $3.94(-3)$ & $3.53(-3)$ & $3.21(-3)$ & $3.07(-3)$ & $2.21(-3)$ & $1.77(-3)$ & 2.2 \\
\hline $2 p^{5} 3 d(3 / 2,5 / 2)_{4}$ & $4.48(-03)$ & $3.99(-03)$ & $3.61(-03)$ & $-03)$ & $2.45(-03)$ & $1.95(-03)$ & \\
\hline & $4.38(-03)$ & $3.91(-03)$ & $3.54(-03)$ & $3.39(-03)$ & $2.42(-03)$ & $1.93(-03)$ & 1.1 \\
\hline $2 \mathrm{p}^{5} 3 \mathrm{~d}(3 / 2,3 / 2)_{2}$ & $3.08(-03)$ & $2.65(-03)$ & $2.33(-03)$ & $2.21(-03)$ & $1.47(-03)$ & $1.13(-03)$ & \\
\hline & $2.93(-03)$ & $2.53(-03)$ & $2.24(-03)$ & $2.12(-03)$ & $1.43(-03)$ & $1.10(-03)$ & 2.5 \\
\hline $2 \mathrm{p}^{5} 3 \mathrm{~d}(3 / 2,5 / 2)_{3}$ & $3.20(-03)$ & $2.85(-03)$ & $2.60(-03)$ & $2.50(-03)$ & $1.97(-03)$ & $1.76(-03)$ & \\
\hline & $3.11(-03)$ & $2.78(-03)$ & $2.54(-03)$ & $2.44(-03)$ & $1.94(-03)$ & $1.74(-03)$ & 1.5 \\
\hline $2 \mathrm{p}^{5} 3 \mathrm{~d}(3 / 2,5 / 2)_{1}$ & $3.37(-02)$ & $3.51(-02)$ & $3.65(-02)$ & $3.72(-02)$ & $4.37(-02)$ & $4.93(-02)$ & \\
\hline & $3.35(-02)$ & $3.49(-02)$ & $3.63(-02)$ & $3.70(-02)$ & $4.36(-02)$ & $4.92(-02)$ & 0.3 \\
\hline $2 \mathrm{p}^{5} 3 \mathrm{~d}(1 / 2,3 / 2)_{2}$ & $2.41(-03)$ & $2.12(-03)$ & $1.91(-03)$ & $1.82(-03)$ & $1.27(-03)$ & $1.00(-03)$ & \\
\hline & $2.33(-03)$ & $2.06(-03)$ & $1.86(-03)$ & $1.77(-03)$ & $1.25(-03)$ & $9.85(-04)$ & 1.7 \\
\hline $2 \mathrm{p}^{5} 3 \mathrm{~d}(1 / 2,5 / 2)_{2}$ & $3.61(-03)$ & $3.20(-03)$ & $2.88(-03)$ & $2.75(-03)$ & $1.93(-03)$ & $1.53(-03)$ & \\
\hline & $3.49(-03)$ & $3.10(-03)$ & $2.80(-03)$ & $2.67(-03)$ & $1.89(-03)$ & $1.50(-03)$ & 1.8 \\
\hline $2 \mathrm{p}^{5} 3 \mathrm{~d}(1 / 2,5 / 2)_{3}$ & $3.12(-03)$ & $2.83(-03)$ & $2.62(-03)$ & $2.54(-03)$ & $2.09(-03)$ & $1.91(-03)$ & \\
\hline & $3.05(-03)$ & $2.77(-03)$ & $2.58(-03)$ & $2.50(-03)$ & $2.07(-03)$ & $1.89(-03)$ & 1.1 \\
\hline $2 \mathrm{p}^{5} 3 \mathrm{~d}(1 / 2,3 / 2)_{1}$ & $3.83(-02)$ & $4.01(-02)$ & $4.19(-02)$ & $4.28(-02)$ & $5.08(-02)$ & $5.76(-02)$ & \\
\hline & $3.82(-02)$ & $4.00(-02)$ & $4.18(-02)$ & $4.27(-02)$ & $5.07(-02)$ & $5.75(-02)$ & 0.1 \\
\hline
\end{tabular}

The Maxwellian excitation rate coefficient from the ground level to level $i$ is given by

$$
Q_{g \rightarrow i}=\int_{0}^{\infty} \sigma_{g \rightarrow i}(E) v f(E) \mathrm{d} E,
$$

where $\sigma_{g \rightarrow i}$ is the excitation cross section, $v$ is the free-electron velocity and $f(E)$ is the Maxwellian energy distribution. $B_{i \rightarrow g}$ is the branching ratio from level $i$ to the ground level versus radiation to all lower levels 


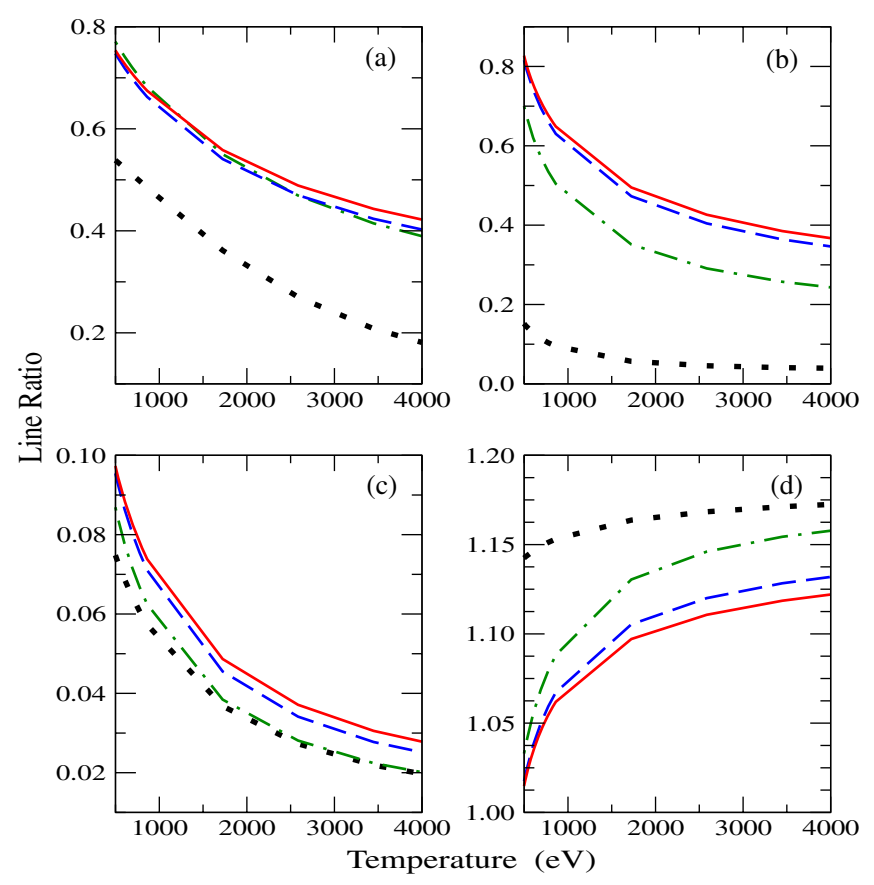

Figure 3. Line-intensity ratios as a function of temperature for various transitions in $\mathrm{Kr}^{26+}$ determined from the 139-level $R$-matrix calculation with radiation damping: (a) the $\mathrm{M} 2 / 3 \mathrm{G}$ line ratio; (b) the $3 \mathrm{E} / 3 \mathrm{C}$ line ratio; (c) the $3 \mathrm{~F} / 3 \mathrm{C}$ line ratio and (d) the $3 \mathrm{C} / 3 \mathrm{D}$ line ratio. All collisional radiative calculations were performed at an electron density of $1.0 \times 10^{14} \mathrm{~cm}^{-3}$. The dotted curves are calculated from equation (1) and are essentially the ratios of Maxwellian rate coefficients; the dot-dashed curves are from collisional radiative modeling that includes all levels through $2 s^{2} 2 p^{5} 3 l$ (lowest 27 levels in table 1); the long dashed curves are from collisional radiative modeling that includes all levels through $2 s^{2} 2 p^{5} 4 l$ (the lowest 75 levels in table 1); the solid curves are from collisional radiative modeling that includes all 139 levels in table 1.

$$
B_{i \rightarrow g}=\frac{A_{i \rightarrow g}}{\sum_{k<i} A_{i \rightarrow k}},
$$

where $A_{i \rightarrow k}$ is the radiative rate from level $i$ to a lower level $k$. For the six transitions given in table 3 , the radiative branching ratios to the ground level are 1.00, with the exception of transition $E$ which has a branching ratio of 0.92 . Thus, the line ratios in this simple approximation are essentially equal to the ratios of the Maxwellian excitation rate coefficients.

However, equation (1) is not adequate for most cases and one must perform collisional-radiative modeling calculations to first determine the population number densities for each level as a function of density and temperature. The intensity line ratios are then given by

$$
\frac{I_{i \rightarrow g}}{I_{j \rightarrow g}}=\frac{N_{i}}{N_{j}} \frac{A_{i \rightarrow g}}{A_{j \rightarrow g}},
$$

where $N_{i}$ is the population number density of level $i$ and $A_{i \rightarrow g}$ is the radiative rate from level $i$ to the ground level. In the collisional-radiative calculations presented here, we employed the isolated-ion approximation so that the population for a given level depends only on radiative decays from that level, radiative cascades from all higher levels, collisional excitation and de-excitation from all other levels to that level, and collisional excitation and de-excitation from that level to all other levels. A more complete model would also include the influence of ionization and recombination between adjacent ions.

Line ratios involving the six transitions listed in table 3 as a function of temperature are presented in figure 3 . They are all calculated at an electron density of $1.0 \times 10^{14} \mathrm{~cm}^{-3}$. We show results determined from equation (1) (dotted curves) and when we included only the lowest 27 levels (dashed-dot curves), the lowest 75 levels (dashed curves) and all 139 levels (solid curves) in the collisional radiative modeling.

We found that these ratios do not change appreciably as a function of density for densities less than approximately $1.0 \times 10^{15} \mathrm{~cm}^{-3}$. Therefore, they cannot be used as density diagnostics for the range of densities present in tokamak plasmas. For the results shown here at a density of $1.0 \times$ $10^{14} \mathrm{~cm}^{-3}$, the level populations thus depend almost entirely on radiative cascades and very little on collisional excitation and de-excitation. As we see from figure 3, the ratios $\mathrm{M} 2 / 3 \mathrm{G}, 3 \mathrm{E} / 3 \mathrm{C}$ and $3 \mathrm{~F} / 3 \mathrm{C}$ should be effective as temperature diagnostics, while variations with temperature for the $3 \mathrm{C} / 3 \mathrm{D}$ line ratio are probably too small for this purpose.

By comparing the dotted curve with the other three curves for the $\mathrm{M} 2 / 3 \mathrm{G}$ ratio shown in (a), it is clear that radiative cascades from the $2 \mathrm{~s}^{2} 2 \mathrm{p}^{5} 3 \mathrm{l}$ levels have a pronounced effect on this particular line ratio, but the effects of cascades from still higher levels are not significant. In the case of the $3 \mathrm{E} / 3 \mathrm{C}$ ratio shown in (b), the radiative cascades included in the 27 level modeling calculation again have a large effect, but now the additional cascades included in the 75-level calculation are also significant, while the effects of cascades from still higher levels are small. This is also true of the $3 \mathrm{C} / 3 \mathrm{D}$ ratio shown in (d), especially at the lower temperatures. Finally, the effects of cascades from the $2 s^{2} 2 p^{5} 3 l$ levels on the $3 F / 3 C$ ratio shown in (c) are small, especially at lower temperatures; however, those from higher levels are more significant. Some additional changes in these ratios would have occurred if we had included levels above the highest level of $2 s^{2} 2 p^{5} 5 l$ configuration; however, these curves indicate that they would be relatively small.

\section{Conclusions}

We have completed the first $R$-matrix calculation on neonlike $\mathrm{Kr}^{26+}$ that includes levels above the highest level of the $2 s^{2} 2 p^{5} 3 l$ configuration. The effects of radiation damping on the resonance contributions to excitation in this ion were investigated and found to be small. All electric dipole, electric quadrupole, magnetic dipole radiative rates and effective collision strengths between all 139 levels have been generated and these data are available on the ORNL CFADC website.

Simplified collisional-radiative modeling on this ion was carried out and intensity line ratios were calculated involving six different transitions used previously in other Ne-like systems. For the range of densities important in tokamak plasmas, these line ratios do not vary with density. However, the effects of radiative cascades on these line ratios are significant and collisional-radiative modeling is required to obtain accurate values. 


\section{Acknowledgments}

This work was partially supported by US DoE grants DE-FG02-99ER54367 to Rollins College and DE-FG0205ER54819 through Auburn University. Most of the computational work was carried out at the National Energy Research Scientific Computing Center in Oakland, California.

\section{References}

[1] Smith B W, Raymond J C, Mann J B and Cowan R D 1985 Astrophys. J. 298898

[2] Doron R and Behar E 2002 Astrophys. J. 574518

[3] Gu M F 2003 Astrophys. J. 5821241

[4] Chen G X 2008 Phys. Rev. A 77022701

[5] Chen G X and Pradhan A K 2002 Phys. Rev. Lett. 89013202

[6] Chen G X and Pradhan A K 2003 J. Phys. B: At. Mol. Opt Phys. 36453

[7] Loch S D, Pindzola M S, Ballance C P and Griffin D C 2006 J. Phys. B: At. Mol. Opt. Phys. 3985

[8] Chen G X 2007 Phys. Rev. A 76062708

[9] Rice J E, Fournier K B, Goetz J A, Marmar E S and Terry J L 2000 J. Phys. B: At. Mol. Opt. Phys. 335435

[10] Bhatia A K, Feldman U and Seely J F 1985 At. Data Nucl. Data Tables 32435

[11] Reed K J 1985 Internal Memo, Lawrence Livermore National Laboratory

[12] Zhang H, Sampson D H, Clark R E H and Mann J B 1987 At. Data Nucl. Data Tables 3717
[13] Gupta G P, Deb N C and Msezane A Z 2000 Phys. Scr. 61175

[14] Dyall K G, Grant I P, Johnson C T, Parpia F A and Plummer E P 1989 Comput. Phys. Commun. 55425

[15] Parpia F A, Froese Fischer C and Grant I P 1996 Comput. Phys. Commun. 94249

[16] Dong C Z, Xie L Y, Fritzsche S and Kato T 2003 Nucl. Instrum. Methods Phys. Res. B 20587

[17] Berengut J C, Dzuba V A, Flambaum V V and Marchenko M V 2004 Phys. Rev. A 70064101

[18] Berengut J C, Flambaum V V and Kozlov M G 2005 Phys. Rev. A 72044501

[19] Berengut J C, Flambaum V V and Kozlov M G 2006 Phys. Rev. A 73012504

[20] Ballance C P and Griffin D C 2006 J. Phys. B: At. Mol. Opt. Phys. 393617

[21] Norrington P H and Grant I P 1987 J. Phys. B: At. Mol. Opt. Phys. 204869

[22] Ait-Tahar S, Grant I P and Norrington N H 1996 Phys. Rev. A 543984

[23] Ballance C P and Griffin D C 2004 J. Phys. B: At. Mol. Opt. Phys. 372943

[24] Mitnik D M, Griffin D C and Badnell N R 2001 J. Phys. B: At. Mol. Opt. Phys. 344455

[25] Mitnik D M, Griffin D C, Ballance C P and Badnell N R 2003 J. Phys. B: At. Mol. Opt. Phys. 36717

[26] Badnell N R, Berrington K A, Summers H P, O'Mullane M G, Whiteford A D and Ballance C P 2004 J. Phys. B: At. Mol. Opt. Phys. 374589

[27] Berrington K A, Ballance C P, Griffin D C and Badnell N R 2005 J. Phys. B: At. Mol. Opt. Phys. 381667

[28] Burgess A 1974 J. Phys. B: At. Mol. Phys. 7 L364 